\title{
Introduction : Évolutions postsuburbaines en Europe et dans le Nouveau Monde
}

Introduction: Post-suburban development in Europe and the New World Einleitung: Post-suburbane Entwicklungen in Europa und der Neuen Welt

\section{Axel Borsdorf}

\section{OpenEdition}

\section{Journals}

Édition électronique

URL : http://journals.openedition.org/rge/245

DOI : $10.4000 /$ rge. 245

ISSN : $2108-6478$

\section{Éditeur}

Association des géographes de l'Est

Édition imprimée

Date de publication : 1 juin 2005

Pagination : 125-132

ISSN : 0035-3213

\section{Référence électronique}

Axel Borsdorf, «Introduction : Évolutions postsuburbaines en Europe et dans le Nouveau Monde», Revue Géographique de l'Est [En ligne], vol. 45 / 3-4 | 2005, mis en ligne le 10 juin 2009, consulté le 08 septembre 2020. URL : http://journals.openedition.org/rge/245 ; DOI : https://doi.org/10.4000/rge.245

Ce document a été généré automatiquement le 8 septembre 2020

Tous droits réservés 


\section{Introduction : Évolutions postsuburbaines en Europe et dans le Nouveau Monde}

Introduction: Post-suburban development in Europe and the New World

Einleitung: Post-suburbane Entwicklungen in Europa und der Neuen Welt

\section{Axel Borsdorf}

\section{La ville en mutation}

1 Trois tableaux d'Albrecht Dürer, un artiste qui, mieux que tout autre maître, a su incarner la mutation intervenue entre le Moyen-Âge et l'époque moderne, entre l'époque gothique et la Renaissance, révèlent le visage de la ville d'Innsbruck aux alentours de 1500. Aujourd'hui, l'impressionnant ensemble gothique de cette ville serait sans doute inscrit à l'inventaire du Patrimoine culturel de l'Humanité, en vue de le préserver pour les générations à venir. Malheureusement, aucun des bâtiments gothiques, que Dürer a su peindre de façon aussi expressive, n'y est plus conservé. Ils ont dû céder la place, déjà peu de temps après, aux splendides constructions de la Renaissance et du Baroque, monuments qui mériteraient également d'être inscrits à l'Inventaire culturel de l'humanité.

2 Cet exemple montre très clairement que chaque ère historique s'exprime à travers son propre langage des formes et qu'elle vise à traduire celui-ci dans le contexte urbain. La ville est mutation, un fait, dont il faudrait tenir compte à tout prix.

Dès lors que l'on considère le paysage humanisé comme une manifestation d'attitudes d'esprit, d'« esprit formé » - "geformten Geist " (Schwind 1962) -, il est possible d'affirmer que les trois décennies écoulées ont été soumises à une mutation tellement profonde que l'on est incité à parler d'un nouveau changement d'ère, celui du passage du moderne au postmoderne (Lyotard 1979, Becker 1996, Borsdorf 2006). La figure 1 tente d'illustrer graphiquement cette mutation. 
4 Cette mutation structurelle et fonctionnelle semble être aussi bouleversante que la "révolution urbaine" du XIX ${ }^{\mathrm{e}}$ siècle, lorsque la dichotomie originelle "ville/milieu rural» s'est dissoute et que les villes commencèrent à s'étendre dans l'espace environnant. Ce fut la fin d'une époque qui a suscité la ville compacte, entourée de murailles, et celle du dicton "la muraille sépare bourgeois et paysans ». Ce fut l'émergence de la continuité ville/espace rural, qui a structuré l'espace urbain comme un ensemble nettement hiérarchisé, dont l'importance devait diminuer au fur et à mesure que l'on s'éloignait du noyau urbain en direction de la périphérie.

5 Le noyau urbain s'appropria toutes les fonctions déterminantes en matière tertiaire (commerce et services), la City se dota encore de quelques fonctions commerciales et industrielles spécialisées, tandis que la couronne extérieure fut essentiellement affectée à la construction de quartiers d'habitation suburbains. Ces derniers se vidaient chaque matin de jour ouvrable, en-dehors des "veuves vertes » demeurées sur place pour s'occuper du ménage et de l'éducation des enfants. Dans ce contexte, "Suburbia » apparaissait alors clairement comme une ville-dortoir, un espace fonctionnant en complémentarité avec le noyau urbain, avec lequel elle était fortement reliée par des flux professionnels, commerciaux et récréatifs.

6 Le modèle de société familiale, selon lequel l'homme vaquait à ses activités professionnelles et l'épouse autant aux besoins ménagers que culinaires, s'étant désagrégé depuis environ trente ans, il en est de même de la structure urbaine. L'aire suburbanisée a déjà été valorisée, dans le cadre du modèle d'organisation de la "ville régionale ", à partir du moment où des fonctions du noyau urbain ont été transférées, dans la foulée de la "concentration décentralisée ", aux carrefours constitués par les gares du réseau express régional ferroviaire et du métro. Toutefois, seules la motorisation de masse qui a apporté la liberté de mobilité à l'ensemble des couches sociales, ainsi que la conquête continue, par les femmes, de nouveaux champs d'activité professionnelle, ont donné le coup de grâce à la ville-dortoir tout autant qu'aux « veuves vertes".

7 Dans les anciens espaces suburbains, mais aussi au-delà de l'ancien périmètre urbain sont nées des structures nouvelles, également en-dehors de la construction de logements. Le commerce a découvert la "prairie verte» ("grüne Wiese ») comme lieu d'implantation attractif pour des unités nécessitant de grandes surfaces de vente : centres commerciaux, " malls » et aires de marchés spécifiques sont apparus du jour au lendemain. La réussite du temple de la consommation à la périphérie urbaine a été fondée sur l'offre attractive, la bonne accessibilité, les parkings gratuits et la mobilité illimitée qui favorise la clientèle.

De nombreux services se sont établis à leur tour à la périphérie urbaine : des cités de bureaux, des universités et d'autres établissements de formation, des cliniques, même des infrastructures administratives municipales. Les «businessparks » modernes favorisent la connexion entre les fonctions commerciales, industrielles et de services. Des aéroports internationaux induisant des fonctions nouvelles ont transformé leur ancien aspect, limité à la seule fonction de transport aérien : ils ont suscité en premier lieu l'émergence d'hôtels et de firmes logistiques, puis l'implantation d'entreprises internationales de prestations de services, ensuite l'aménagement de «malls» et, en fin de compte, la mise en place de fonctions récréatives. 
Cette mutation interroge une nouvelle dimension du développement spatial le long de l'ancien périmètre urbain : depuis belle lurette, celui-ci n'est plus exclusivement le "poumon vert» de la ville, ainsi que le territoire d'action de la soi-disant « détente tranquille ». Si ce sont plutôt des discothèques et des «night clubs» qui font encore partie des infrastructures traditionnelles de cet espace, les innovations portent sur l'arrivée de centres sportifs et récréatifs, de terrains de golf, de "country clubs", d'arènes de « cross country ".

10 Finalement, la culture est à son tour «exurbanisante» en ce qui concerne des initiatives culturelles, des maisons d'art, théâtres et événements saisonniers marquants. On a presque l'impression que l'avant-garde culturelle est localisée à présent sur les aires extérieures de la ville et que l'innovation s'y décide, une innovation que les équipements en centre-ville peinent à suivre.

11 En Allemagne, Thomas Sieverts (1997) a forgé le terme de "ville intermédiaire" («Zwischenstadt») pour désigner cette nouvelle constellation spatiale, qu'il définit comme étant localisée « entre un lieu et l'orbite mondiale, l'espace et le temps, la ville et le monde rural ». Axel Priebs (2001) a recours au terme " postsuburbia », suivant en cela les auteurs nord-américains (Kling, Olin, Poster 1991, 1995) ; en Suisse, Baccini \& Oswald (1998) ont trouvé le terme " ville en réseau » (« Netzstadt»), tandis qu'Eisinger \& Schneider (2003) ont parlé d'« urbanscape » et de « milieu rurbain («Stadtland»); en France Piron \& Dubois-Taine (1998) ont eu recours à « ville émergente ». Il y a un quart de siècle déjà, Moewes (1985) a observé la formation de cette nouvelle structure, qu'il a appelée "groupement ville-campagne ». La figure 2 modélise celle-ci. Les flèches y symbolisent l'intensité des articulations migratoires.

12 Il importe d'affirmer que des évolutions urbaines sont pour la première fois présentées de manière globale. La mutation de la suburbanisation en posturbanisation concerne aujourd'hui la plupart des grandes villes du globe. Il est vrai que les structures de base de celles-ci reflètent encore les modèles traditionnels de leurs espaces culturels respectifs; les processus modernes de croissance sont toutefois soumis aux mêmes mécanismes ; aussi suscitent-ils des formes et structures analogues, comme c'est le cas également en Europe. Les travaux de Severino Escolano, Rodrigo Hidalgo et Christoph Stadel, publiés dans la présente livraison de la Revue géographique de l'Est, illustrent clairement ce phénomène.

\section{De la suburbanisation à la postsuburbanisation}

13 Pour bien comprendre la notion de postsuburbanisation, il importe de préciser au préalable celle de suburbanisation. L'apparition de quartiers suburbains en Europe était liée aux causes suivantes : ce fut d'abord un processus de croissance simple, lorsqu'au $\mathrm{XIX}^{\mathrm{e}}$ siècle les enceintes de villes, gênantes pour la rapide montée de la population urbaine, furent démolies, sous la pression de l'explosion démographique suscitée par l'exode rural. Comparés à la vieille ville, les nouveaux quartiers n'apportèrent pas d'amélioration de la qualité de vie: les tristes lotissements de maisons en bande britanniques, les casernes locatives aux arrière-cours surbâties, sur le continent européen, furent les premières illustrations de la suburbanisation. Cette image devait cependant évoluer au début du XXe siècle, lorsque Ebenezer Howard, en Angleterre, et, placé dans une ambiance d'autres prémisses idéologiques, Theodor Fritsch, en Allemagne, rendirent les unités d'habitation suburbaines bien plus attractives. Le 
«Bauhaus» et d'autres architectes modernes améliorèrent la qualité résidentielle fonctionnelle; de nouvelles technologies de construction (notamment le préfabriqué et la construction en béton) rendirent les bungalows et les logements en pleine propriété accessibles à une large part de la population. Depuis les années 1960, la phase de couverture des besoins résidentiels a été ainsi remplacée par celle relative à l'incitation à l'ouverture résidentielle. Cette sollicitation a été confortée par l'apparition et la diffusion croissante de périodiques illustrés, spécialement destinés à la publicité faite autour des nouvelles façons d'habiter: tels "Schöner wohnen" (Hamburg), «Zuhause » (Hamburg)..., en Allemagne, «Maison française » (Paris), « Maison Magazine » (Chantilly), « Toute la maison » (Paris)..., en France.

Depuis cette réorientation, le déménagement en banlieue a été souvent motivé par le souci de venir habiter « au vert » et dans une ambiance ouverte, calme, au bon air. Le prix du foncier relativement modéré en périphérie urbaine, ainsi que, fréquemment, la bonne accessibilité des transports en commun (une réussite du modèle d'aménagement en ville régionale!), facilitèrent la réalisation du rêve de vivre au vert. La plupart du temps, les nouvelles villes suburbaines étaient constituées de jeunes familles à revenus moyens, dont les personnes actives participaient aux mouvements de migration quotidienne vers les villes-centres et les zones industrielles.

$\mathrm{Au}$ fil des années, cette structure spatiale a révélé toutefois, de plus en plus, ses inconvénients. Son

architecture monotone, associée à des matériaux de construction de faible qualité (plaques de béton non insonorisées), à des parcs stériles et à un manque d'infrastructures de première nécessité, a déclenché des problèmes psychiques, non seulement auprès des "veuves vertes ", mais aussi parmi les jeunes. Le vandalisme, la drogue et, de moins en moins rarement, la fuite vers des zones à problèmes en centreville («Les enfants de la gare Zoo » à Berlin, dont le titre en allemand est « Die Kinder vom Bahnhof Zoo ») étaient des indicateurs significatifs du « caractère inhospitalier de nos villes » («Unwirtlichkeit unserer Städte »), selon le terme auquel Mitscherlich eut recours en 1970.

Mais quelle est donc maintenant l'innovation qui justifie l'introduction d'un nouveau terme pour caractériser l'évolution de la périphérie urbaine ? Le bien-être croissant, le besoin grandissant en espace résidentiel individuel, la pénurie en superficie résidentielle dans les centres-villes, ainsi que le renforcement de la compétition entre des communes à démographie stagnante, en vue de gagner des habitants supplémentaires, ont modifié la structure fonctionnelle entre la ville et la campagne. La population actuelle des quartiers périphériques comporte aussi des personnes seules, des couples sans enfants, des retraités, de nouvelles catégories d'habitants relevant de toutes les couches de la population; aux maisons monofamiliales ont été ajoutés des ensembles résidentiels collectifs.

Il est étonnant que la direction des flux migratoires ait changé également : naguère les trains de banlieue en direction du centre étaient bondés, mais vides en sens inverse ; actuellement, ils transportent à peu près autant de voyageurs dans les deux sens. La ville de Vienne nous fournit des statistiques éloquentes à ce sujet : ici, à peine $12 \%$ des actifs nourrissent encore les flux quotidiens en direction de la ville-centre, contre $20 \%$ en sens inverse.

La cause de ce changement réside dans le fait que la périphérie offre toujours davantage d'emplois. Elle est également dotée de centres commerciaux, de "malls", 
d'aires de marchés spécifiques et de centres de foires, ainsi que d'équipements du secteur de la formation et de la santé. Cette nouvelle donne a fait perdre aux quartiers périphériques leur ancienne fonction de complémentarité par rapport au centre-ville; ceux-ci ont accédé eux-mêmes à la fonction de centralité. La population vit en aire postsuburbaine, une aire qui les fait bénéficier de sa centralité : les habitants $\mathrm{y}$ couvrent leurs besoins et $\mathrm{y}$ travaillent; en fin de compte, ils y vivent aussi leurs loisirs.

La tristesse architecturale et paysagère de jadis est à présent remplacée par une grande diversité de styles et une nouvelle approche résidentielle, de nature écologique, associée à un aménagement écologique des espaces verts. L'émergence d'une offre diversifiée et satisfaisante d'équipements de loisirs rend inutile l'ancienne évasion hebdomadaire au vert ( $c f$. tableau 1, en ce qui concerne la comparaison entre le suburbain et le postsuburbain). La périphérie donne l'occasion de vivre pleinement des styles de vie pluriels, de s'adonner à des activités sportives variées, de béné ficier d'un approvisionnement attractif, en relation avec des possibilités de participer à des événements à forte émotion; elle permet aussi de se réaliser professionnellement dans une ambiance de travail agréable.

Tableau 1 : Comparaison entre la suburbanisation et la postsuburbanisation.

\begin{tabular}{|c|c|c|}
\hline Caractéristiques & Suburbanisation & Postsuburbanisation \\
\hline Impulsion & Croissance démographique & $\begin{array}{l}\text { Cas du rideau de fer, immigration en } \\
\text { provenance de l'Est et du Sud vers les } \\
\text { noyaux urbains, mobilité individuelle }\end{array}$ \\
\hline Conditions & $\begin{array}{l}\text { Demande en logements, en } \\
\text { équipements industriels, en } \\
\text { niveaux de prix faibles, en } \\
\text { accessibilité aux transports publics }\end{array}$ & $\begin{array}{l}\text { "Transformation ", globalisation, } \\
\text { changement social, diversité des styles de } \\
\text { vie, ségrégation, polarisation, } \\
\text { flexibilisation post-fordiste, motorisation }\end{array}$ \\
\hline Image & $\begin{array}{l}\text { Existence dans un cadre naturel, à } \\
\text { l'écart de la pollution }\end{array}$ & $\begin{array}{l}\text { Styles de vie, (post) modernité, sportivité, } \\
\text { cadre propice aux loisirs }\end{array}$ \\
\hline $\begin{array}{l}\text { (Infra) } \\
\text { structure }\end{array}$ & $\begin{array}{l}\text { Seulement l'infrastructure de base, } \\
\text { seulement un peu d'emploi de } \\
\text { niveau élaboré }\end{array}$ & $\begin{array}{l}\text { Infrastructure spécialisée, niveau d'emploi } \\
\text { élaboré, commerce }\end{array}$ \\
\hline $\begin{array}{l}\text { Formes et } \\
\text { structure }\end{array}$ & $\begin{array}{l}\text { Architecture pauvre et espaces } \\
\text { verts }\end{array}$ & $\begin{array}{l}\text { Architecture diversifiée et espaces verts, } \\
\text { mais des « ufos » et des « clones» }\end{array}$ \\
\hline Acteurs & $\begin{array}{l}\text { Classes modestes et moyennes, } \\
\text { familles jeunes }\end{array}$ & $\begin{array}{l}\text { Toutes les classes, investisseurs, hommes } \\
\text { d'affaires, « développeurs » sportifs }\end{array}$ \\
\hline Résultats & $\begin{array}{l}\text { Tours en béton, cités-dortoirs, } \\
\text { « veuves vertes », monotonie }\end{array}$ & $\begin{array}{l}\text { Fragmentation sociale, économique et } \\
\text { spatiale, multifonctionalité, transformation } \\
\text { de l'espace rural, déclin de la centralité } \\
\text { dans les lieux centraux }\end{array}$ \\
\hline
\end{tabular}


\begin{tabular}{|l|l|l|}
\hline Fonction & Complémentarité de la ville-centre & Zones indépendantes, ni urbaines ni rurales \\
\hline
\end{tabular}

Source : Borsdorf 2004, p. 12.

20 La dynamique postsuburbaine a bénéficié d'autres impulsions en provenance de la demande résidentielle de la part d'immigrés qui ont jeté leur dévolu sur les centresvilles. Cette intrusion a conduit de nombreux habitants du centre-ville à s'établir dans les quartiers périphériques, tant par suite d'une motivation positive que négative.

De petits «clones» sont certes apparus dans les centres-villes. Mais une certaine spécialisation fonctionnelle s'est dessinée plutôt dans les communes périphériques : certaines communes ont bénéficié de l'établissement d'équipements commerciaux de niveau élevé, d'autres de «businessparks » dotés d'entreprises de services, d'autres encore de l'arrivée d'un secteur de formation, d'un centre de foire, d'un aéroport, d'universités privées, de cliniques. Ainsi sont nés de véritables archipels urbains, reliés entre eux par des routes et des flux virtuels de communication (figure 1).

Il importe d'insister sur le fait que ces structures n'ont pas été suscitées par la mise en œuvre de concepts clairement définis ou par des plans d'aménagement. Elles sont plutôt le résultat non programmé d'une mutation du système sociétal, politique, économique et culturel, qui a somme toute encore accéléré le mouvement en cours : le retrait de l'État de ses responsabilités économiques et sociétales s'est opéré à travers la dérégulation, qui a réduit l'importance tant de la planification que de l'aménagement du territoire. Ce changement politico-économique est appelé "transformation", tandis que le terme de mondialisation recouvre l'orientation vers des modèles libéraux et l'irruption de la mondialisation.

Il est toutefois judicieux de parler également d'un changement d'époque, c'est-à-dire du passage de l'époque moderne à l'époque postmoderne (Wood 2003, Watson / Gibson 1995, Soja 2000, Borsdorf 2004). De multiples caractéristiques attribuées généralement à l'époque postmoderne (Borsdorf 2006) sont aussi le propre de l'époque postsuburbaine. 
Figure 1 : L'archipel urbain.

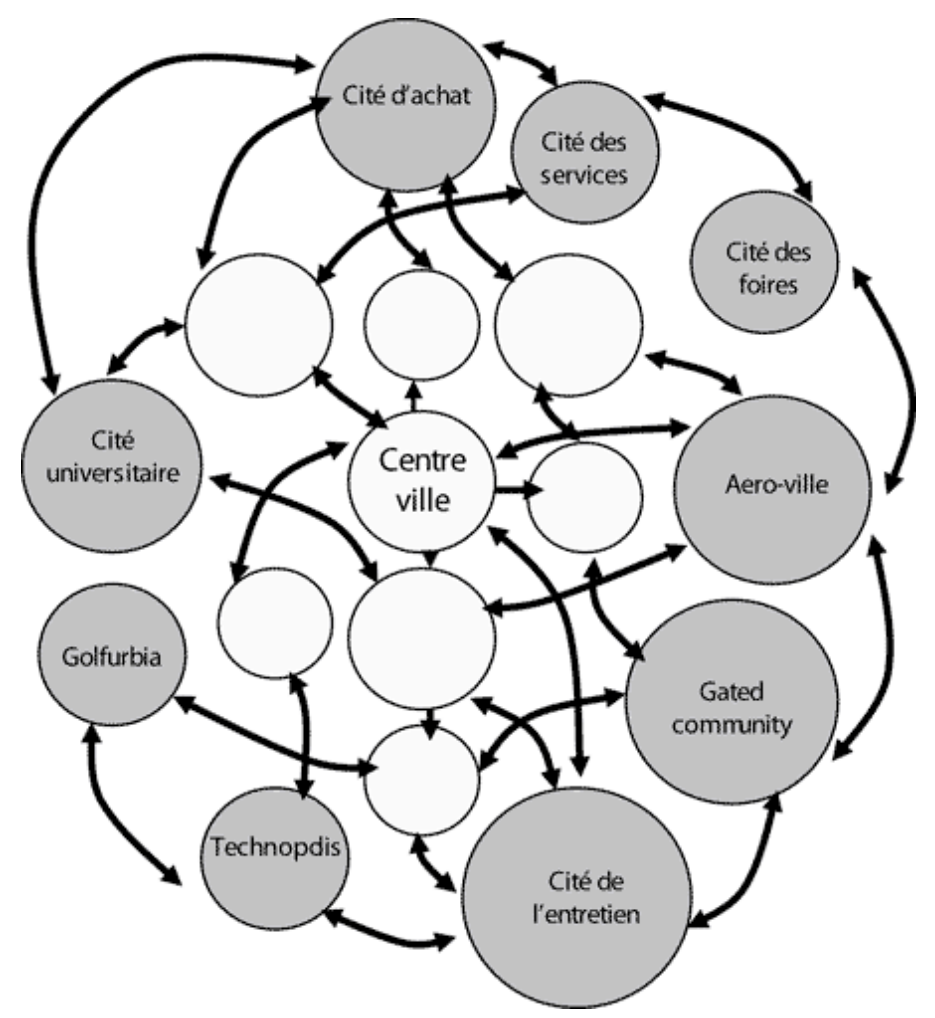

Selon Kunzmann 2001, sensiblement modifié.

Figure 2 : Modèle du paysage urbain postmoderne.

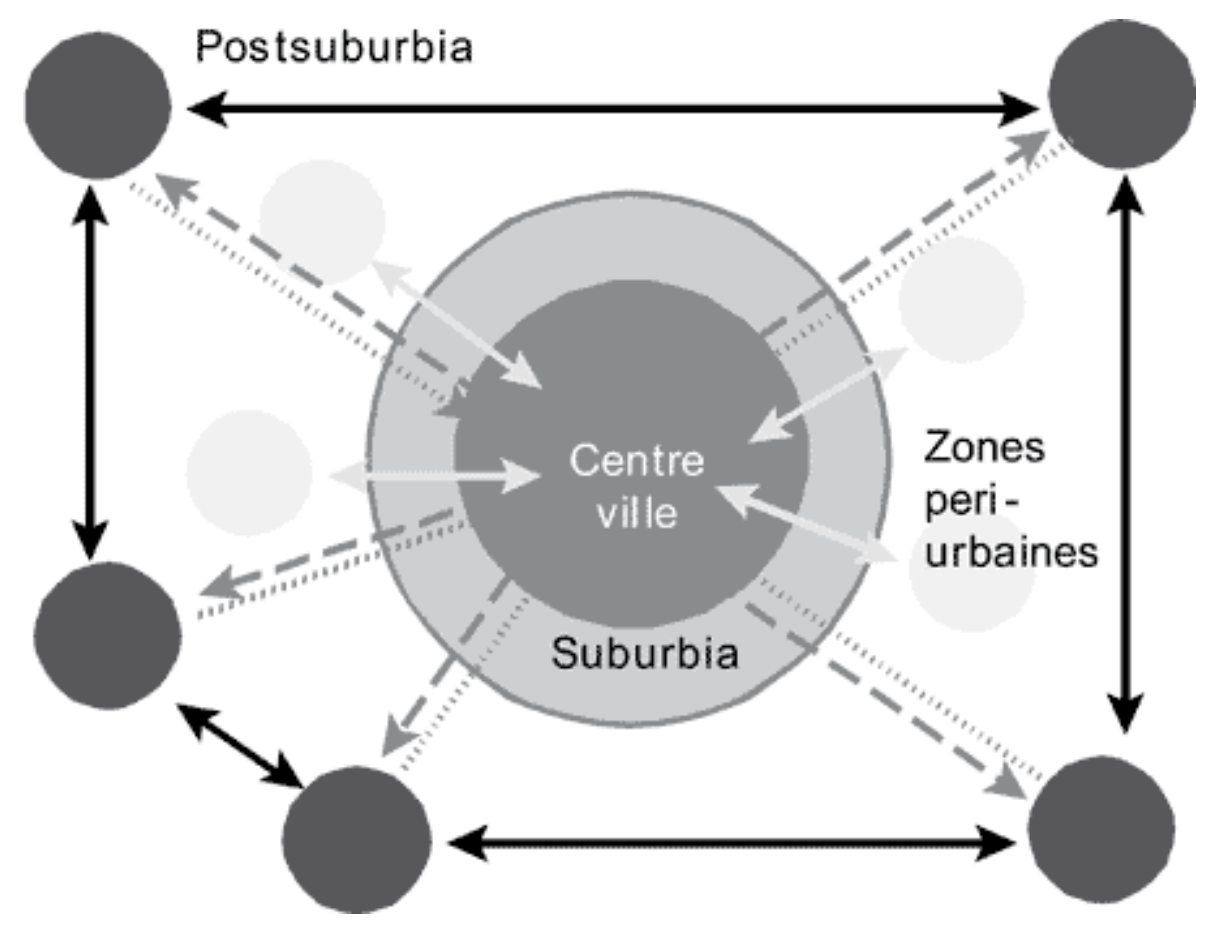

Source : Borsdorf 2004, p. 10. 


\section{L'état des lieux}

théorisation. Elles sont traitées par Burdack (2005), Borsdorf et al. (2004). En matière méthodologique, Burdack distingue la perspective fonctionnaliste, structuraliste et culturelle. La démarche fonctionnaliste s'appuie sur des projections d'écologie urbaine de l'Ecole de Chicago; elle se fonde, en fin de compte, sur des modèles d'équilibre économiques. Des concurrences et positions socio-spatiales (van der Berg 1999), ainsi que des modèles de rente foncière (Häussermann 1997) conduisent par là même à des structures spatiales transformées. Par contraste, les approches structuralistes ont comme point de départ des prémisses de déséquilibre. En conséquence, les structures spatiales ne sont pas le résultat de processus de marché quasi- "naturels ", mais de conflits sociétaux (Harvey 1985). La perspective culturo-spatiale a contré initialement la thèse de l'uniformisation globale et insiste sur les évolutions urbaines spécifiques en matière culturelle. Aujourd'hui, cependant, les transformations sont perçues également, de façon croissante, sous l'angle de la mondialisation (Borsdorf 2004, Borsdorf/Hidalgo 2004).

En dépit de la diversité des investigations, des études comparées ont fait défaut jusqu'en 2004. Cette lacune est à présent comblée, par suite de la publication des recherches de deux vastes projets consacrés, dans un premier temps, à l'Europe : celui, dirigé par Joachim Burdack, dans le cadre de la "Deutsche Forschungsgemeinschaft » (DFG), orienté vers des périphéries métropolitaines européennes, appuyé sur des études de cas portant sur Berlin, Budapest, Madrid, Moscou, Paris et la Randstad-Holland; il s'est penché sur les nouveaux pôles économiques et les pôles de restructuration. Les 
résultats obtenus ont donné lieu à la publication d'un résumé comparatif (Burdack/ Herfert/Rudolph 2005).

L'autre projet induit par l'Union européenne, appelé «Outskirts of European Cities ", coordonné par Geneviève Dubois-Taine) a mobilisé 25 chercheurs relevant de 15 Etats différents. Sans exclure nécessairement des études de cas, il a visé une approche comparée renforcée à l'échelle internationale et l'établissement d'une typologie, invitant les groupes de travail à étudier trois finalités : la structuration, la dynamique et la gouvernance.

Le troisième axe de recherche a été également partie prenante des projets communautaires européens. 16 partenaires répartis dans six Etats ont été chargés de se pencher sur le thème, appelé "Competitive Metropolises" (coordonné par Axel Borsdorf), du développement des périphéries métropolitaines, sous l'angle de la compétitivité. Il est intéressant de constater que ce projet a analysé, parallèlement à celui coordonné par Burdack sur les pôles de restructuration, et indépendamment de lui, ce qui est appelé les "large projects». La publication des résultats de ces recherches est prévue pour 2006.

Le quatrième et dernier axe d'investigation est destiné à parachever la vaste recherche métropolitaine européenne. Il est orienté vers la comparaison globale. Le présent cahier de la Revue géographique de l'Est est appelé à introduire cette étude.

Peter Görgl analyse ce phénomène à partir de trois études de cas portant sur l'espace ambiant de la capitale autrichienne, Vienne. Il part du constat que, dans chacun de ces cas, l'évolution vers la postsuburbanisation est partie de conditions différentes les unes des autres, soit : des friches industrielles, des lieux d'implantation déjà existants, mais dont les potentialités ont été revalorisées, puis d'anciennes aires agricoles. Ce point de départ différencié a donné lieu aussi à de nouvelles structures différenciées: un « patchwork » de centres technologiques, de rencontre et de loisirs, appelé à valoriser le milieu suburbain de telle façon qu'il peut s'appeler désormais postsuburbain; la revitalisation et la valorisation tant sociale qu'infrastructurelle d'anciens quartiers suburbains dans une commune-dortoir typique ; la création de toutes pièces d'un parc résidentiel de luxe postmoderne, ressemblant à une "gated community" nordaméricaine, construit, pour ainsi dire, sur la "prairie verte", doté d'une lagune artificielle, d'un «Clubhouse » et de nombreuses infrastructures récréatives.

Pierre Frankhauser étudie d'un point de vue géométrique les formes et structures de la périphérie urbaine. Il met l'accent sur l'émergence, sur ce type de territoire, d'un modèle spatial fractal; il présente en outre une méthode de mesure du degré de fractalisation.

Jean-Marie Halleux se penche sur le rôle de l'économie foncière et immobilière dans le façonnement des périphéries urbaines en Belgique. Il s'appuie sur une démarche de Krätke (1991), portant sur l'observation de la place des innovations, s'intéressant en l'occurrence à celle des acteurs du nouveau développement. Il apparaît que l'analyse des types de comportement et des motivations est bien plus instructive pour la compréhension du processus de restructuration que la simple description des nouvelles unités spatiales issues de la mutation. De manière intéressante, les conclusions de cette recherche vont à l'encontre de ce qu'affirment maints représentants de la théorie du comportement, qui rêvent déjà d'une "Géographie sans espace " (Werlen 1993), 
mettant l'accent sur la grande importance des structures spatiales en place dans les décisions prises par les instances concernées.

En Espagne, le phénomène de la suburbanisation est relativement nouveau. Celle-ci n'a débuté çà et là qu'à la fin de la dictature de Franco, dans les années 1980, en tant que "suburbanisation de rattrapage ». Le processus de la suburbanisation s'est mélangé, presque insensiblement, avec des phénomènes de postsuburbanisation, de telle façon que ces deux tendances constituent une synthèse, qui fait défaut ailleurs, entre l'évolution de l'urbanisme moderne et celle de l'urbanisme postmoderne. Rainer Wehrhahn étudie ce processus à partir de l'exemple offert par la capitale espagnole, Madrid, en tenant compte du rôle des subventions communautaires européennes et de celui des acteurs locaux dans les rapports entre les articulations réciproques $d u$ « global » et du « local».

Les structures para-urbaines de nombreux paysages touristiques sont connues depuis longtemps. Il s'agit d'agglomérations comportant des résidences secondaires. Christoph Stadel analyse de telles structures au Manitoba et aboutit à la conclusion selon laquelle ces villes se caractérisent par de nombreuses structures postsuburbaines. La coutume, selon laquelle on passe le week-end ou les vacances estivales dans un chalet au bord d'un lac, est répandue depuis longtemps au Canada, de façon analogue à celle propre à la Scandinavie. Dans le cas particulier, il peut s'agir de véritables agglomérations de résidences secondaires, ressemblant à des lotissements de maisons monofamiliales suburbaines, quoique dépourvues, en tant que quartiers suburbains, du support d'un centre-ville. Le commerce de détail de proximité du lieu de résidence principal des migrants est en effet suffisant pour un séjour de courte durée. Stadel pose alors la question de savoir si de tels phénomènes ne sont encore que para-urbains ou se présentent déjà comme postsuburbains.

La fragmentation socio-économique et fonctionnelle des ensembles urbains est un phénomène mondial, qui donne lieu à des recherches intenses depuis quelques décennies. Elle concerne tout particulièrement des villes d'Amérique latine, dans lesquelles la fragmentation par barrages de rues, quartiers résidentiels clôturés, veillés jour et nuit, ainsi que d'autres formes de ségrégation extrême sont partout présentes, même visuellement. Severino Escolano et Jorge Ortiz examinent ce phénomène sur la base d'hypothèses vérifiables par des méthodes relevant de systèmes d'information géographique très affinés. Ils constatent que tous les processus de fragmentation sont connectés les uns aux autres. La grande ville latino-américaine - en l'occurrence Santiago du Chili - apparaît comme un archipel urbain dont les quartiers périphériques comportent des formes postsuburbaines expressives.

Rodrigo Hidalgo part de cette constatation pour étendre l'étude à l'ensemble de la diffusion, à Santiago du Chili comme dans son espace ambiant, du phénomène résidentiel ségrégationniste ; il inclut dans son investigation les villes nouvelles de plus de 50000 habitants entièrement clôturées. La question se pose de savoir si les excès du processus de fragmentation et de ségrégation portent encore les caractéristiques de la postsuburbanisation ou s'ils sont déjà marqués éventuellement par l'ère posturbaine. Une telle évolution est susceptible de demeurer propre à un espace culturel déterminé. Des réalisations urbanistiques d'ampleur comparable sont en effet surtout perceptibles en Amérique latine (Alphaville : Coy \& Pöhler 2002 ; Nordelta : Janoschka 2002 ; Santiago du Chili : Borsdorf/Hidalgo 2005). Le projet d'une implantation de cette 
nature, dans le style d'Alphaville, aux environs de Lisbonne, montre toutefois que l'Europe n'est pas épargnée par ce phénomène.

\section{BIBLIOGRAPHIE}

ARING J. (1999). - Suburbia - Postsuburbia - Zwischenstadt. Die jüngere Wohnsiedlungsentwicklung im Umland der großen Städte Westdeutschlands und Folgerungen für die regionale Planung und Steuerung. Arbeitsmaterial für Raumforschung und Landesplanung 262. Hannover.

ARING J. (2001). - Postsuburbia - Herausforderungen für stadtregionales Management. In : AG der Regionalverbändein Ballungsräumen und Kommunalverband Großraum Hannover (eds.) : Postuburbia - Herausforderungen für stadtregionales Management. Beiträge zur regionalen Entwicklung 90, Hannover, p. 115-124.

BACCINI P. \& OSWALD F. (ed.) (1998). - Netzstadt. Transdisziplinäre Methoden zum Umbau urbaner Systeme. Zürich.

BECKER J. (1996). - Geographie in der Postmoderne ? Zur Kritik postmodernen Denkens in Stadtforschung und Geographie. Potsdamer Geographische Forschungen 12. Potsdam.

BORSDORF A. (2003). - Desde la dicotomía a la unión campo-ciudad. From dichotomy to the union city-countryside. In : Luzón J.L., Vila J. \& Rubio F. : La delimitación del Área Metropolitana de Barcelona aplicando el método NUREC. Barcelona's Metropolitan Area delimitation by the NUREC method. Barcelona, p. 11-18.

BORSDORF A. (2004). - Wenn Stadt « geformter Geist » ist, wofür steht dann Postsuburbia? Spurenlesen im ruralen Raum. In : Borsdorf A. \& Mayer v. (Hg.), 2004 : Konvergenz und Divergenz der Kulturen in den Randzonen der Städte.Beiträge der Sektion 3.7. Internationale Konferenz Das Verbindende der Kulturen. Schriftenreihe Wohnwesen Umland Wien 2, Wien, S. 17-25. BORSDORF A. (2004). - Innsbruck : From city to cyta ? Outskirt development as an indicator of spatial, economic and social development. In : Dubois-Taine G. (Hg.) : From Helsinki to Nicosia. Eleven case studies \& synthesis. European Cities. Insights on Outskirts. Paris, S. 75-96.

BORSDORF A. (2004). - Commercial areas in the outskirts of European cities. Locations and structures. In : Borsdorf A. \& Zembri P. (Hg.), 2004 :Structures. European Cities : Insights on Outskirts. Paris. S. 129-148.

BORSDORF A. (2004). - On the way to post-suburbia ? Changing structures in the outskirts of Euopean cities. In : Borsdorf A.\& Zembri P. (Hg.), 2004 :Structures. European Cities : Insights on Outskirts. Paris, S. 7-30.

BORSDORF A. (2006). - Geographische Gesellschaften in der Postmoderne. Festschrift zum 150 jährigen Bestehen der Österreichischen Geographischen Gesellschaft (im Druck).

BORSDORF A. \& HIDALGOR. (2005). - Städtebauliche Megaprojekte im Umland lateinamerikanischer Städte - eine Antithese zur Stadt? In : Geographische Rundschau 57, 10, p. 30-38. 
BORSDORF A. \& SCHÖFFTHALER G. (2000). - Erzeugen Einkaufszentren Verkehr ? Eine Bewertung des Einkaufsverkehrs am Beispiel nicht-integrierter Einkaufszentren im Großraum Innsbruck. - In : Erdkunde 132, 2000, 2, p. 148-156.

BORSDORF A. \& ZEMBRI P. (eds.) (2004). - Structures. European Cities - Insights on Outskirts. Paris. BORSDORF A., CRIVELLI S., DEMETRIOU C., FRANKHAUSER P., GIOVANNINI P., KLASANDER A.-J., KOCH M., SCHUMACHER M., ZEMBRI P. (2004). - Parallels and differences in the outskirts of European cities. A. methodological reflection and a comparative matrix. In : Borsdorf A. \& Zembri P. (Hg.), 2004 : Structures. European Cities : Insights on Outskirts. Paris, S. 169-181.

BRAKE K., DANGSCHAT J.S., HERFERTG. (eds.) (2001). - Suburbanisierung in Deutschland. Aktuelle Tendenzen.Opladen.

BURDACK J. (2001). - Die städtische Peripherie zwischen " suburbanen » und « postsuburbanen » Entwicklungen. In : Berichte zur deutschen Landeskunde 75, 2/3, p. 188-196.

BURDACK J. (2005). - Die metropolitane Peripherie zwischen suburbanen und postsuburbanen Entwicklungen. In : Burdack J., Herfert G. \& Rudolph R. (eds.) 2005 : Europäische metropolitane Peripherien. Beiträge zur Regionalen Geographie 61, Leipzig.

BURDACK J. \& HERFERT G. (1998). - Neue Entwicklungen an der Peripherie europäischer Großstädte. In : Europa Regional 6, 2, p. 26-44.

BURDACK J., HERFERT G., RUDOLPHR. (eds.) (2005). - Europäische metropolitane Peripherien. Beiträge zur Regionalen Geographie 61, Leipzig.

COY M. \& PÖHLER M. (2002). - Condominios fechados und die Fragmentierung der brasilianischen Stadt. Typen, Akteure, Folgewirkungen. In : Geographica Helvetica57 (4), S. 264-277.

DECROLY J.M., DESSOUROUX C., VAN CRIEKINGEN M. (2001). - Les dynamiques contemporaines de privatisation des espaces urbains dans les villes européennes. In : Belgeo 1, p. 3-19.

DÉZERT B. (1991). - Les nouvelles formes d'activité économique périurbaine. In : Dézert B., Metton A. \& Steinberg J. (eds.) : La périurbanisation en France. Paris.

DOLLEN B.V.D. (1983). - Standrandphänomene. In : DIE ERDE 114, p. 69-71.

DUBoIS-TAINE G. (ed.) (2004). - From Helsinki to Nicosia. Eleven Case Studies \& Synthesis. European Cities - Insights on Outskirts. Paris.

EISINGER A. \& SCHNEIDER M. (eds.) (2003). - Stadtland Schweiz. Untersuchungen und Fallstudien zur räumlichen Struktur und Entwicklung in der Schweiz. Basel, Boston, Berlin.

HÄUSSERMANN H. (1997). - Von der kapitalistischen zur sozialistischen Stadt. In : Kovács Z. \& Wiesner R. (eds.) : Prozesse und Perspektiven der Stadtentwicklung in Ostmitteleuropa. Münchner Geographische Hefte 76, Passau, p. 21-31.

HILDALGO R., TRUMPER R., BORSDORFA. (eds.) (2004). - Transformaciones urbanas y procesos territoriales. Lecturas del nuevo dibujo de la ciudad latinoamericana. GEOlibro. Santiago de Chile.

FоOт м. (2000). - The urban periphery, myth and reality. Milan 1950-1990. In : Cities 4, 1, p. 7-26. FRANZÉN M. \& HALLEUXJ.-M. (eds.) (2004). - Dynamics. European Cities - Insights on Outskirts. Paris. HARVEY D. (1985). - The Urbanization of Capital. Oxford. 
HATZFELD U. (2001). - Freizeitsuburbanisierung. - Löst sich die Freizeit aus der Stadt ? In : Brake K., Dangschat J.S. \& Herfert G. (eds.), 2001 : Suburbanisierung in Deutschland. Aktuelle Tendenzen. Opladen, p. 81-95.

HESSE M. (2001). - Mobilität und Verkehr in (Post) Suburbia. Ein Ausblick. In : RaumPlanung 95, p. 65-69.

JANOSCHKA M. (2002). - Wohlstand hinter Mauern. ISR Forschungsberichte 28, Wien : ÖAW.

KIRBY A. (2003). - City and Country. In : Cities 20, 2, p. 75-76.

KLING R., OLIN S., POSTERM. (eds.) (1991). - The emergence of Postsuburbia : An Introduction. Postsuburban California : the Transformation of Postwar Orange County since World War II. Berkely, Los Angeles, Oxford. $2^{\text {nd }}$ edition 1995.

KRAKOVER S. \& BORSDORF A. (2000). - Spatial Dynamics of Urban Expansion. The Case of Innsbruck, Austria. In : DIE ERDE 131, 2, p. 120-161.

KRÄTKE S. (1991). - Strukturwandel der Städte. Städtesystem und Grundstücksmarkt in der postfordistischen Ära.Frankfurt/M.

KUNZMANN K.K. (2001). - Welche Zukünfte für Suburbia? Acht Inseln im Archipel der Stadtregion. In : Brake K., Dangschat J.S. \& Herfert G. (eds.), 2001 : Suburbanisierung in Deutschland. Aktuelle Tendenzen. Opladen, p. 213-221.

LYOTARD J.F. (1979). - La conditione postmoderne. Rapport sur le savoir. Paris : Passagen.

MC ELDOWNEY M. (ed.) (2004). - Governance. European Cities - Insights on Outskirts. Paris.

MITSCHERLICH A. (1970). - Die Unwirtlichkeit unserer Städte. Anstiftung zum Unfrieden. 9th ed.

Frankfurt/M.

MOEWES W. (1980). - Grundfragen der Lebensraumgestaltung: Raum und Mensch, Prognose, offene planung und Leitbild. Berlin.

MOEWES W. (1981). - Stadt-Land-Verbund in der Planungspraxis : am Beispiel des städtebaulichen Rahmenplans Gießen-Wetzlar. Berlin.

MOULAERT F. \& SWYNGEDOUWE. (1990). - Regionale Entwicklung und die Geographie flexibler Produktionssysteme. Theoretische Auseinandersetzungen und empirische Belege aus WestEuropa und den USA. In : Borst U. et al. (eds.) : Das neue Gesicht der Städte. Basel, p. 89-108.

PÉRON R. (2001). - The political management of change in urban retailing. In : International Journal of Urban and Regional Research 25, 4, p. 847-878.

PIRON O. \& DUBOIS-TAINE G. (1998). - La Ville émergente. Constats pour renouveler les lignes d'action publiques. Paris.

PRIEBS A. (2001). - Postsuburbia - Herausforderungen für stadtregionales Management. In : AG der Regionalverbändein Ballungsräumen und Kommunalverband Großraum Hannover (eds.) : Postsuburbia - Herausforderungen für stadtregionales Management. Beiträge zur regionalen Entwicklung 90, Hannover, p. 7-11.

SCHULER M., PERLIK M., PASCHE N. (2004). - Nicht-städtisch, rural oder peripher - wo steht der ländliche Raum heute? ARE, Bundesamt für Raumentwicklung, Bern.

SCHWIND M. (1962). - Kulturlandschaft als geformter Geist. Darmstadt.

SIEVERTS T. (1997). - Zwischenstadt. Zwischen Ort und Welt, Raum und Zeit, Stadt und Land. Bauwelt Fundamente 113. Braunschweig, Wiesbaden. 
SOJA E.W. (2000). - Postmetropolis. Oxford.

VAN DEN BERG L. (1999). - The urban life cycle and the role of a market-oriented revitalization policy in Western Europe. In : summers A., Cheshire P.C. \& Senn L. (eds.) : Urban Change in the United States and Western Europe.Washington D.C., p. 539-558.

WATSON S. \& GIBSONK. (eds.) (1995). - Postmodern Cities and Spaces. Oxford.

WEICHHART P. (2005). - Zukunftsaufgabe Kooperation. - Stöckl W. (ed.) : Metropolis versus Peripherie. Die Herausforderung Kooperation. EUREGIO Regionalkonferenz 2005, Wien, p. 3-7.

WERLEN B. (1993). - Gibt es eine Geographie ohne Raum ? Zum Verhältnis von traditioneller Geographie und zeitgenössischen Gesellschaften. In : Erdkunde 47, 4, S. 241-255.

wooD G. (2003). - Die postmoderne Stadt : Neue Formen der Urbanität im Übergang vom zweiten ins dritte Jahrtausend. In : Gebhardt H., Reuber P. \& Wolkersdorfer G. (eds.) : Kulturgeographie. Aktuelle Ansätze und Entwicklungen. Heidelberg, Berlin, p. 131-147.

\section{AUTEUR}

\section{AXEL BORSDORF}

Institut für Geographie der Leopold-Franzens-Universität, Innrain 52, A-6020 Innsbruck 